\title{
Pancreatic cancer - lessons from the past decade
}

Pancreatic ductal adenocarcinoma (PDAC) is an on-going challenge in oncology, both for the physicians, and even more for the patients. In PDAC, we are confronted with a cancer that, although not very frequent, is perceived as unavoidably lethal, with a 5-year survival rate of $5 \%$, ranking presently fourth in cancer-associated mortality, largely due to its rapid spread in the asymptomatic phase of the disease. ${ }^{[1]}$ Undoubtedly, this frightening lethality of PDAC has at the same time paved the path for massive clinical and basic science efforts to tailor more effective therapy regimens or to at least improve the quality of life of PDAC patients. However, until date, the attained progress, when compared with the considerable amount of efforts, remains minute.

This marginality of success is best reflected in a comparison of overall cancer incidence, PDAC incidence, and the proportion of deaths to the diagnosed PDAC cases in the last decade. In the past 10 years, the overall cancer incidence in, e.g., the USA has increased by $21 \%$, whereas the PDAC incidence has risen by $44 \%{ }^{\left[{ }^{11}\right.}$ In a comparison of a number of deaths due to PDAC to its incidence, in 2012 , this proportion equaled $85.1 \%$ and holds at $85.3 \%$ in 2014. ${ }^{[1]}$ Hence, the incidence of PDAC rises at a higher rate than the overall cancer incidence, whereas its lethality remains constant and very high.

Nonetheless, the proportion of PDAC-associated lethality to PDAC incidence in 2006 was $96 \%$, thus $10 \%$ greater than in 2014. ${ }^{[1]}$ Even though this difference may be due to the improved reporting of PDAC cases to cancer registers in the US, it encourages us to believe that the continuing efforts to improve the prognosis of PDAC may be yielding their fruits.

The central problem, that is, our inability to diagnose PDAC is an early phase, has not yet been overcome. No single month goes by without at least one publication on potential novel biomarkers of PDAC. The more we understand the biology of PDAC, its interactions with the host, and the changes in the metabolic behavior of PDAC, the more

\begin{tabular}{|l|l|}
\hline \multicolumn{2}{|c|}{ Access this article online } \\
\hline Quick Response Code: & Website: \\
\hline & www.jmpo.org \\
\hline
\end{tabular}

molecular alterations are detected to happen in early phases of tumorigenesis. However, the proportion of markers that eventually find access into the clinical routine remains also very low, largely due to the insufficient sensitivity or specificity, that is, their low accuracy for diagnosing early PDAC. In the era of molecular, personalized medicine, the sole serum marker that we can use for monitoring the disease course and for estimating the prognosis remains CA19-9, which, however, does also not aid in the early diagnosis of PDAC. ${ }^{[2]}$ Therefore, from the perspective of serum biomarkers, there is a persistent need for specific markers of early disease that can be used for screening purposes.

The other option for early diagnosis, that is, sensitive, specific and high resolution imaging modalities, may be a valuable, though costly, alternative. Indeed, current cost-effectiveness and cost-benefit calculations do not demonstrate sufficient benefit for routine computed tomography (CT) and magnetic resonance (MR)-based screening. Hence, we need a modality without too high costs and with sufficient accuracy for the early PDAC lesions. In this direction, a promising technical development was fluorescence-endoscopy of pancreatic intraepithelial neoplasia in experimental murine PDAC ${ }^{[3]}$ It is imaginable that, once validated in the human setting, such an endoscopic screening may enter the routine clinical practice for detecting PDAC precursor lesions.

One issue that has not found sufficient resonance in the context of PDAC screening is the association between PDAC and the occurrence of diabetes mellitus. This link has been addressed in studies from the US where the researchers investigated the frequency of diabetes mellitus specifically in early PDAC. Based on their observations, they postulated that patients with PDAC can be identified early by new-onset diabetes mellitus as the latter is a harbinger of PDAC development, increased rates of perioperative complications and worse long-term prognosis. As screening for diabetes mellitus is not costly per se, the option of exposing patients with new-onset diabetes diagnosed during routine diabetes screening to subsequent imaging for PDAC may be an acceptable strategy of PDAC screening.

The single most important factor for the prognosis of patients with resectable PDAC remains the resection status, that is, the possibility of achieving a microscopically complete resection of the tumor with tumor-free margins 
(R0 status). Looking at the developments of the past decade in the management of PDAC, one can quickly recognize that all efforts, including those of the surgeon, an oncologist, and the pathologist, are toward holding this premise. Our surgical strategy has become more "aggressive," since we learned that we do not need to refrain from performing venous resections and multivisceral resections to achieve R0 status. Indeed, when performed by experienced hands within large-volume centers, venous and multivisceral R0 resections have been convincingly shown to be associated with a comparable overall survival as $\mathrm{R} 0$ resections in which no vein or neighboring organ infiltration was observed. ${ }^{[4,5]}$ In the case of arterial infiltration, we recognized that the peri-and post-operative mortality inflicted on the patients due to the arterial resection impairs the overall survival, thus rendering arterial resections, although technically feasible, mostly ineffective in the management of PDAC. Similarly, although extended lymphadenectomy has been initially favored by studies with selected patient's cohorts from the Far East, large-scale prospective randomized multicenter trials have demonstrated no benefit for extended lymphadenectomy when compared to standard lymphadenectomy in achieving improved survival or higher R0 rates. ${ }^{[6]}$ However, we also learned that increasing age per $s e$, in the absence of significant co-morbidity, should not be a contraindication to the surgery of R0-resectable PDAC. ${ }^{[7]}$ Therefore, in the past years, high quality prospective studies delivered considerable evidence on our "boundaries" in managing PDAC, on how far we can, may and should go when trying to achieve the $\mathrm{R} 0$ status.

The major contribution of the oncology toward achieving the R0 status might be neoadjuvant therapy in PDAC. Subsequent to the success of 5-fluorouracil, oxaliplatin, irinotecan, and leucovorin-based neoadjuvant therapy in enabling R0-resectable PDAC in the initially "borderline"resectable PDAC, ${ }^{[8]}$ increasing number of clinical trials have been initiated to attain and to detect the best possible preoperative shrinkage of the tumor volume in borderlineresectable PDAC. ${ }^{[9]}$ The problem remains that PDAC has low response rates to all neoadjuvant treatment regimens and therefore presently, neoadjuvant downstaging is more serving to select better treatment-responsive cases than real downstaging of the local disease. Owing to these studies, we know that such neoadjuvant therapy regimens are linked to a comparable overall survival rate after resection as primarily resected PDAC cases. Importantly, the close imaging-based monitoring of these cases once again confirmed that what we see on imaging for PDAC, e.g., on $\mathrm{CT}$ or magnetic resonance imaging scans, may not reflect the true extent of the disease. Indeed, lack of a measurable shrinkage of the primary tumor volume has been shown to be still associated with resectable disease as the initially tumor-invaded tissue areas are progressively replaced by desmoplastic scar tissue during neoadjuvant therapy. Intensified efforts to perform a greater number of randomized clinical studies are needed to make progress.

A recent important step toward enhanced comparability of neoadjuvant therapy outcomes in PDAC has been the introduction of definitions of borderline-resectable PDAC. ${ }^{[10]}$ However, there are still some discrepancies in the criteria of the two most commonly applied definitions, which is why the interpretation of the outcomes of trials of the coming years may turn problematic. ${ }^{[10]}$ At this stage in which we sensed the major advantage associated with neoadjuvantly treatable PDAC, efforts to unify these definitions in an internationally accepted single definition are urgently needed.

Though, similar efforts are yet necessary to standardize the histopathological assessment of PDAC specimens. In the absence of a standardized processing of the resected tumor and of a standard reporting scheme of the resection status, the outcomes of different studies and surgical centers are not going to be comparable. In the UK, where a standardized analysis of PDAC specimens was already introduced years ago, major discrepancies have been reported to exist among pathologists in the reporting of the R-status, e.g., which resection plane they take into account for assessing the R-status and how many millimeters between the tumor cells and the resection margin they consider to define R-status. ${ }^{[1]}$ Another aspect of this problem is that in the USA, the definition and allocation of R1 status considerably varies from the Europe-wide R-status classification. ${ }^{[12]}$ In the absence of unified reporting and assessment systems for the PDAC tumor specimens, it is no wonder that retrospective analyses of PDAC specimens reveal higher than previously reported $\mathrm{R} 1$ cases. On the other hand, there are also visible efforts toward standardizing tumor specimen processing and reporting, but these efforts have not yet been sufficiently transmitted and promoted to international expert audiences in other continents.

What holds considerable promise in improving the prognosis of PDAC are preclinical studies in which previously unknown, very peculiar aspects of tumor-host interactions are elucidated. That the outcome of such studies can be rapidly translatable into the clinical practice is exemplified by the currently increased administration of nab-paclitaxel in advanced PDAC. Subsequent to reports on increased concentrations of albumin receptors in PDAC stroma, researchers first administered albuminbound paclitaxel in preclinical studies with considerable success in murine PDAC. ${ }^{[13]}$ Out of the observations made by these studies, nab-paclitaxel as an albumin-bound 
chemotherapeutic has soon been tested in advanced PDAC and is currently accepted as a superior alternative to gemcitabine alone in the palliative setting. ${ }^{[14]}$

Two very promising areas of preclinical research deserve attention for foreseeing future clinical efforts to improve the prognosis of PDAC. Following the 2009 study on the improved chemotherapeutic drug delivery after depletion of the stroma in murine PDAC via a Hedgehog signaling inhibitor, a similar compound was tested in the clinical setting, and the trial had to be stopped due to the increased lethality of the group of patients who received the stromadepleting agent. ${ }^{[15]}$ The reasons for this disappointment associated with stroma depletion in PDAC were recently reported by two concomitant studies in which stroma depletion in PDAC was shown to be associated with the occurrence of more anaplastic and invasive tumors. ${ }^{[1,17]}$ Hence, stroma is currently perceived not only as a barrier to drug delivery, but rather as a defense mechanism of the tumor microenvironment to limit tumor spread. However, further studies underlined that in contrast with whole stroma depletion, depletion of selected components of the tumor stroma like hyaluronic acid or collagen may have contrasting effects on tumor growth. Indeed, owing to the preclinical success of a pegylated hyaluronic acid degrading enzyme (peg-hyaluronidase) in murine PDAC, a current clinical trial reported improved preliminary outcome of patents who took this enzyme in addition to chemotherapy. ${ }^{[18]}$ On the other hand, a recent study that investigated the metabolomic-molecular profile of recurrent or chemotherapy-resistant PDAC cells found that these surviving cancer cells are very much dependent on oxidative phosphorylation and not that much on glycolysis as their ancestor cancer cell clones. ${ }^{[19]}$ Accordingly, administration of inhibitors of oxidative phosphorylation in these preclinical models entailed a dramatic improvement in the prognosis of murine PDAC. ${ }^{[19]}$ Therefore, we may well await the results of clinical trials using selective stroma-targeting agents and inhibitors of oxidative phosphorylation in the coming years.

The multitude of both clinical and preclinical advances in the management of PDAC especially in the past 5-years suggests on the one hand that we are dealing with a complex cancer, driven by multiple tumor-intrinsic and extrinsic factors, which, in this extent, are rarely encountered in other malignancies. On the other hand, though, these very recent advances also give enough reason to believe that there may be much more than just minute improvement in the prognosis of PDAC in the very near future. However, based on the lessons from the past decade, we recognized that this improvement will be possible if surgeons, oncologists, pathologists and basic scientists perceive themselves in close and well-communicating partners in tackling PDAC.
Ihsan Ekin Demir, Helmut Friess

Department of Surgery, Klinikum Rechts Der Isar, Technische Universität München, Munich, Germany.

E-mail: helmut.friess@tum.de

\section{REFERENCES}

1. Siegel R, Ma J, Zou Z, Jemal A. Cancer statistics, 2014. CA Cancer J Clin 2014;64:9-29.

2. Bauer TM, El-Rayes BF, Li X, Hammad N, Philip PA, Shields AF, et al. Carbohydrate antigen $19-9$ is a prognostic and predictive biomarker in patients with advanced pancreatic cancer who receive gemcitabine-containing chemotherapy: A pooled analysis of 6 prospective trials. Cancer 2013;119:285-92.

3. Eser S, Messer M, Eser P, von Werder A, Seidler B, Bajbouj $\mathrm{M}$, et al. In vivo diagnosis of murine pancreatic intraepithelial neoplasia and early-stage pancreatic cancer by molecular imaging. Proc Natl Acad Sci U S A 2011;108:9945-50.

4. Hartwig W, Hackert T, Hinz U, Hassenpflug M, Strobel O, Büchler MW, et al. Multivisceral resection for pancreatic malignancies: Risk-analysis and long-term outcome. Ann Surg 2009;250:81-7.

5. Riediger H, Makowiec F, Fischer E, Adam U, Hopt UT. Postoperative morbidity and long-term survival after pancreaticoduodenectomy with superior mesenterico-portal vein resection. J Gastrointest Surg 2006;10:1106-15.

6. Jang JY, Kang MJ, Heo JS, Choi SH, Choi DW, Park SJ, et al. A prospective randomized controlled study comparing outcomes of standard resection and extended resection, including dissection of the nerve plexus and various lymph nodes, in patients with pancreatic head cancer. Ann Surg 2014;259:656-64.

7. Kanda $M$, Fujii $T$, Suenaga $M$, Takami $H$, Inokawa $Y$, Yamada S, et al. Pancreatoduodenectomy with portal vein resection is feasible and potentially beneficial for elderly patients with pancreatic cancer. Pancreas 2014;43:951-8.

8. Gourgou-Bourgade S, Bascoul-Mollevi C, Desseigne F, Ychou M, Bouché $\mathrm{O}$, Guimbaud $\mathrm{R}$, et al. Impact of FOLFIRINOX compared with gemcitabine on quality of life in patients with metastatic pancreatic cancer: Results from the PRODIGE 4/ACCORD 11 randomized trial. J Clin Oncol 2013;31:23-9.

9. Fong ZV, Tan WP, Lavu $H$, Kennedy EP, Mitchell DG, Koniaris LG, et al. Preoperative imaging for resectable periampullary cancer: Clinicopathologic implications of reported radiographic findings. J Gastrointest Surg 2013;17:1098-106.

10. Katz MH, Marsh R, Herman JM, Shi Q, Collison E, Venook $A P$, et al. Borderline resectable pancreatic cancer: Need for standardization and methods for optimal clinical trial design. Ann Surg Oncol 2013;20:2787-95.

11. Feakins R, Campbell F, Verbeke CS. Survey of UK histopathologists' approach to the reporting of resection specimens for carcinomas of the pancreatic head. J Clin Pathol 2013;66:715-7.

12. Verbeke CS, Leitch D, Menon KV, McMahon MJ, Guillou PJ, Anthoney $\mathrm{A}$. Redefining the $\mathrm{R} 1$ resection in pancreatic cancer. Br J Surg 2006;93:1232-7.

13. Neesse A, Michl P, Tuveson DA, Ellenrieder V. nab-Paclitaxel: Novel clinical and experimental evidence in pancreatic cancer. Z Gastroenterol 2014;52:360-6.

14. Von Hoff DD, Ervin T, Arena FP, Chiorean EG, Infante J, Moore $\mathrm{M}$, et al. Increased survival in pancreatic cancer with nab-paclitaxel plus gemcitabine. $N$ Engl J Med 2013;369:1691-703. 
15. Olive KP, Jacobetz MA, Davidson CJ, Gopinathan A, Mclntyre D, Honess D, et al. Inhibition of Hedgehog signaling enhances delivery of chemotherapy in a mouse model of pancreatic cancer. Science 2009;324:1457-61.

16. Özdemir BC, Pentcheva-Hoang $T$, Carstens JL, Zheng $X, \mathrm{Wu}$ CC, Simpson TR, et al. Depletion of carcinoma-associated fibroblasts and fibrosis induces immunosuppression and accelerates pancreas cancer with reduced survival. Cancer Cell 2014;25:719-34.

17. Rhim AD, Oberstein PE, Thomas DH, Mirek ET, Palermo CF, Sastra SA, et al. Stromal elements act to restrain, rather than support, pancreatic ductal adenocarcinoma. Cancer Cell 2014;25:735-47.
18. Provenzano PP, Hingorani SR. Hyaluronan, fluid pressure, and stromal resistance in pancreas cancer. $\mathrm{Br} \mathrm{J}$ Cancer 2013;108:1-8.

19. Viale A, Pettazzoni $P$, Lyssiotis CA, Ying $H$, Sánchez $N$, Marchesini $\mathrm{M}$, et al. Oncogene ablation-resistant pancreatic cancer cells depend on mitochondrial function. Nature 2014;514:628-32.

How to cite this article: Demir IE, Friess $\mathrm{H}$. Pancreatic cancer - lessons from the past decade. Indian J Med Paediatr Oncol 2015;36:73-6.

Source of Support: Nil, Conflict of Interest: None declared.

\section{"QUICK RESPONSE CODE" LINK FOR FULL TEXT ARTICLES}

The journal issue has a unique new feature for reaching to the journal's website without typing a single letter. Each article on its first page has a "Quick Response Code". Using any mobile or other hand-held device with camera and GPRS/other internet source, one can reach to the full text of that particular article on the journal's website. Start a QR-code reading software (see list of free applications from http://tinyurl.com/yzlh2tc) and point the camera to the QR-code printed in the journal. It will automatically take you to the HTML full text of that article. One can also use a desktop or laptop with web camera for similar functionality. See http://tinyurl.com/2bw7fn3 or http://tinyurl.com/3ysr3me for the free applications. 